\title{
Some normality criteria of functions related a Hayman conjecture
}

\author{
Wenjun Yuan ${ }^{1 *}$, Bing Zhu ${ }^{2}$ and Jianming $\operatorname{Lin}^{3^{*}}$
}

\author{
* Correspondence: \\ wjyuan1957@126.com; \\ ljmguanli@21cn.com \\ 'School of Mathematics and \\ Information Science, Guangzhou \\ University, Guangzhou 510006, \\ China \\ ${ }^{3}$ School of Economic and \\ Management, Guangzhou \\ University of Chinese Medicine, \\ Guangzhou 510006, China \\ Full list of author information is \\ available at the end of the article
}

\begin{abstract}
In the article, we study the normality of families of meromorphic functions

concerning shared values. We consider whether a family meromorphic functions $\mathcal{F}$ is normal in $D$, if for every pair of functions $f$ and $g$ in $\mathcal{F}, f^{n} f$ and $g^{n} g^{\prime}$ share a nonzero value $a$. Two examples show that the conditions in our results are best possible in a sense.
\end{abstract}

\section{Introduction and main results}

Let $f(z)$ and $g(z)$ be two nonconstant meromorphic functions in a domain $D \subseteq \mathbf{C}$, and let $a$ be a finite complex value. We say that $f$ and $g$ share $a$ CM (or IM) in $D$ provided that $f$ - $a$ and $g-a$ have the same zeros counting (or ignoring) multiplicity in $D$. When $a=\infty$ the zeros of $f$ - $a$ means the poles of $f$ (see [1]). It is assumed that the reader is familiar with the standard notations and the basic results of Nevanlinna's value-distribution theory [1-4].

Bloch's principle [5] states that every condition which reduces a meromorphic function in the plane $\mathbf{C}$ to be a constant forces a family of meromorphic functions in a domain $D$ normal. Although the principle is false in general (see [6]), many authors proved normality criterion for families of meromorphic functions corresponding to Liouville-Picard type theorem (see [4]).

It is also more interesting to find normality criteria from the point of view of shared values. In this area, Schwick [7] first proved an interesting result that a family of meromorphic functions in a domain is normal if in which every function shares three distinct finite complex numbers with its first derivative. And later, more results about normality criteria concerning shared values have emerged, for instance, (see [8-10]). In recent years, this subject has attracted the attention of many researchers worldwide.

We now first introduce a normality criterion related to a Hayman normal conjecture [11].

Theorem 1.1 Let $\mathcal{F}$ be a meromorphic function family on domain $D, n L \mathbf{N}$. If each function $f(z)$ of family $\mathcal{F}$ satisfies $f^{n}(z) f(z) \neq 1$, then $\mathcal{F}$ is normal in $D$.

The proof of Theorem 1.1 is because of Gu [12] for $n \geq 3$, Pang [13] for $n=2$, Chen and Fang [14] for $n=1$. In 2004, by the ideas of shared values, Fang and Zalcman [15] obtained:

Theorem 1.2 Let $\mathcal{F}$ be a family of meromorphic functions in $D, n$ be a positive integer. If for each pair of functions $f$ and $g$ in $\mathcal{F}, f$ and $g$ share the value 0 and $f^{n} f$ and $g^{n}$ $g$ share a nonzero value $a$ in $D$, then $\mathcal{F}$ is normal in $D$.

(c) 2011 Yuan et al; licensee Springer. This is an Open Access article distributed under the terms of the Creative Commons Attribution License (http://creativecommons.org/licenses/by/2.0), which permits unrestricted use, distribution, and reproduction in any medium, provided the original work is properly cited. 
In 2008, Zhang [10] obtained a criterion for normality of $\mathcal{F}$ in terms of the multiplicities of the zeros and poles of the functions in $\mathcal{F}$ and use it to improve Theorem 1.2 as follows.

Theorem 1.3 Let $\mathcal{F}$ be a family of meromorphic functions in $D$ satisfying that all zeros and poles of $f \in \mathcal{F}$ have multiplicities at least 3. If for each pair of functions $f$ and $g$ in $\mathcal{F}, f^{\prime}$ and $g^{\prime}$ share a nonzero value $a$ in $D$, then $\mathcal{F}$ is normal in $D$.

Theorem 1.4 Let $\mathcal{F}$ be a family of meromorphic functions in $D, n$ be a positive integer. If $n \geq 2$ and for each pair of functions $f$ and $g$ in $\mathcal{F}, f^{n} f$ and $g^{n} g^{\prime}$ share a nonzero value a in $D$, then $\mathcal{F}$ is normal in $D$.

Zhang [10] gave the following example to show that Theorem 1.4 is not true when $n=$ 1 , and therefore the condition $n \geq 1$ is best possible.

Example 1.1 The family of holomorphic functions $\mathcal{F}=\left\{f_{j}(z)=\sqrt{j}\left(z+\frac{1}{j}\right): j=1,2, \ldots,\right\}$ is not normal in $D=\{z:|z|<1\}$ This is deduced by $f_{j}^{\#}(0)=\frac{j \sqrt{j}}{j+1} \rightarrow \infty$, as $j \rightarrow \infty$ and Marty's criterion [2], although for any $f_{j}(z) \in \mathcal{F}, f_{j} f_{j}^{\prime}=j z+1$. Hence, for each pair $m, j, f_{m} f_{m}^{\prime}$ and $f_{j} f_{j}^{\prime}$ share the value 1 .

Here $f^{\sharp}(\xi)$ denotes the spherical derivative

$$
f^{\#}(\xi)=\frac{\left|f^{\prime}(\xi)\right|}{1+|f(\xi)|^{2}} .
$$

In this article, we will improve Theorem 1.3 and use it to consider Theorem 1.4 when $n=1$. Our main results are as follows:

Theorem 1.5 Let $\mathcal{F}$ be a family of meromorphic functions in $D$ satisfying that all zeros of $f \in \mathcal{F}$ have multiplicities at least 4 and all poles of $f \in \mathcal{F}$ are multiple. If for each pair of functions $f$ and $g$ in $\mathcal{F}, f^{\prime}$ and $g^{\prime}$ share a nonzero value $a$ in $D$, then $\mathcal{F}$ is normal in $D$.

Theorem 1.6 Let $\mathcal{F}$ be a family of meromorphic functions in D satisfying that all zeros of $f \in \mathcal{F}$ are multiple. If for each pair of functions $f$ and $g$ in $\mathcal{F}, f f$ and gg' share a nonzero value a in $D$, then $\mathcal{F}$ is normal in $D$.

Since normality of families of $\mathcal{F}$ and $\mathcal{F}^{*}=\left\{\frac{1}{f} \mid f \in \mathcal{F}\right\}$ is the same by the famous Marty's criterion, we obtain the following criteria from above results.

Theorem 1.7 Let $\mathcal{F}$ be a family of meromorphic functions in $D, n$ be a positive integer. If $n \geq 4$ and for each pair of functions $f$ and $g$ in $\mathcal{F}, f^{-n} f^{\prime}$ and $g^{-n} g^{\prime}$ share a nonzero value $a$ in $D$, then $\mathcal{F}$ is normal in $D$.

Theorem 1.8 Let $\mathcal{F}$ be a family of meromorphic functions in $D$ satisfying that all poles of $f \in \mathcal{F}$ are multiple. If for each pair of functions $f$ and $g$ in $\mathcal{F}, f^{-3} f^{\prime}$ and $g^{-3} g^{\prime}$ share a nonzero value a in $D$, then $\mathcal{F}$ is normal in $D$.

Theorem 1.9 Let $\mathcal{F}$ be a family of meromorphic functions in D satisfying that all zeros and poles of $f \in \mathcal{F}$ have multiplicities at least 3. If for each pair of functions $f$ and $g$ in $\mathcal{F}, f^{-2} f$ and $g^{-2} g^{\prime}$ share a nonzero value a in $D$, then $\mathcal{F}$ is normal in $D$.

Theorem 1.10 Let $\mathcal{F}$ be a family of meromorphic functions in $D$ satisfying that all poles of $f \in \mathcal{F}$ have multiplicities at least 4 and all zeros of $f \in \mathcal{F}$ are multiple. If for each pair of functions $f$ and $g$ in $\mathcal{F}, f^{-2} f$ and $g^{-2} g^{\prime}$ share a nonzero value $a$ in $D$, then $\mathcal{F}$ is normal in $D$.

Example 1.2 The family of holomorphic functions $\mathcal{F}=\left\{f_{j}(z)=j e^{z}-j-1: j=1,2, \ldots,\right\}$ is not normal in $D=\{z:|z|<1\}$ This is deduced by $f_{j}^{\#}(0)=j \rightarrow \infty$, as $j \rightarrow \infty$ and Marty's 
criterion [2], although for any $f_{j}(z) \in \mathcal{F}, \frac{f_{j}^{\prime}}{f_{j}}=1+\frac{j+1}{j e^{z}-j-1} \neq 1$. Hence, for each pair $m, j$, $\frac{f_{j}^{\prime}}{f_{j}}$ and $\frac{f_{j}^{\prime}}{f_{j}}$ share the value 1 .

Remark 1.11 Example 1.1 shows that the condition that all zeros of $f \in \mathcal{F}$ are multiple in Theorem 1.6 is best possible. Both Examples 1.1 and 1.2 show that above results are best possible in a sense.

\section{Preliminary lemmas}

To prove our result, we need the following lemmas. The first is the extended version of Zalcman's [16] concerning normal families.

Lemma 2.1 [17]Let $\mathcal{F}$ be a family of meromorphic functions on the unit disc satisfying all zeros of functions in $\mathcal{F}$ have multiplicity $\geq p$ and all poles of functions in $\mathcal{F}$ have multiplicity $\geq q$. Let $\alpha$ be a real number satisfying $-q<\alpha<p$. Then, $\mathcal{F}$ is not normal at 0 if and only if there exist

(a) a number $0<r<1$;

(b) points $z_{n}$ with $\left|z_{n}\right|<r$;

(c) functions $f_{n} \in \mathcal{F}$;

(d) positive numbers $\rho_{n} \rightarrow 0$

such that $g_{n}(\zeta):=\rho^{-\alpha} f_{n}\left(z_{n}+\rho_{n} \zeta\right)$ converges spherically uniformly on each compact subset of $\mathbf{C}$ to a nonconstant meromorphic function $g(\zeta)$, whose all zeros of functions in $\mathcal{F}$ have multiplicity $\geq p$ and all poles of functions in $\mathcal{F}$ have multiplicity $\geq q$ and order is at most 2.

Remark 2.2 If $\mathcal{F}$ is a family of holomorphic functions on the unit disc in Lemma 2.1, then $g(\zeta)$ is a nonconstant entire function whose order is at most 1 .

The order of $g$ is defined using Nevanlinna's characteristic function $T(r, g)$ :

$$
\rho(g)=\lim _{r \rightarrow \infty} \sup \frac{\log T(r, g)}{\log r} .
$$

Lemma 2.3 [18] or [19] Let $f(z)$ be a meromorphic function and $c \in \mathbf{C} \backslash\{0\}$. If $f(z)$ has neither simple zero nor simple pole, and $f(z) \neq c$, then $f(z)$ is constant.

Lemma 2.4 [20] Let $f(z)$ be a transcendental meromorphic function of finite order in $\mathrm{C}$, and have no simple zero, then $f(z)$ assumes every nonzero finite value infinitely often.

\section{Proof of the results}

Proof of Theorem 1.5 Suppose that $\mathcal{F}$ is not normal in $D$. Then, there exists at least one point $z_{0}$ such that $\mathcal{F}$ is not normal at the point $z_{0}$. Without loss of generality, we assume that $z_{0}=0$. By Lemma 2.1, there exist points $z_{j} \rightarrow 0$, positive numbers $\rho_{j} \rightarrow 0$ and functions $f_{j} \in \mathcal{F}$ such that

$$
g_{j}(\xi)=\rho_{j}^{-1} f_{j}\left(z_{j}+\rho_{j} \xi\right) \Rightarrow g(\xi)
$$

locally uniformly with respect to the spherical metric, where $g$ is a nonconstant meromorphic function in $\mathbf{C}$ satisfying all its zeros have multiplicities at least 4 and all its poles are multiple. Moreover, the order of $g$ is $\leq 2$. 
From (3.1), we know

$$
g_{j}^{\prime}(\xi)=f_{j}^{\prime}\left(z_{j}+\rho_{j} \xi\right) \Rightarrow g^{\prime}(\xi)
$$

and

$$
\begin{gathered}
{f^{\prime}}_{j}\left(z_{j}+\rho_{j} \xi\right)-a=g_{j}^{\prime}(\xi)-a \\
\Rightarrow g^{\prime}(\xi)-a
\end{gathered}
$$

also locally uniformly with respect to the spherical metric.

If $g^{\prime}-a \equiv 0$, then $g \equiv a \xi+c$, where $c$ is a constant. This contradicts with $g$ satisfying all its zeros have multiplicities at least 4. Hence, $g^{\prime}-a \otimes 0$.

If $g^{\prime}-a \neq 0$, by Lemma 2.3, then $g$ is also a constant which is a contradiction with $g$ being not any constant. Hence, $g^{\prime}-a$ is a nonconstant meromorphic function and has at least one zero.

Next, we prove that $g^{\prime}-a$ has just a unique zero. By contraries, let $\xi_{0}$ and $\xi_{0}^{*}$ be two distinct zeros of $g^{\prime}-a$, and choose $\delta(>0)$ small enough such that $D\left(\xi_{0}, \delta\right) \cap D\left(\xi_{0}^{*}, \delta\right)=\phi$ where $D\left(\xi_{0}, \delta\right)=\left\{\xi:\left|\xi-\xi_{0}\right|<\delta\right\}$ and $D\left(\xi_{0}^{*}, \delta\right)=\left\{\xi:\left|\xi-\xi_{0}^{*}\right|<\delta\right\}$. From (3.2), by Hurwitz's theorem, there exist points $\xi_{j} \in D\left(\xi_{0}, \delta\right), \xi_{j}^{*} \in D\left(\xi_{0}^{*}, \delta\right)$ such that for sufficiently large $j$

$$
f_{j}^{\prime}\left(z_{j}+\rho_{j} \xi_{j}\right)-a=0, \quad f_{j}^{\prime}\left(z_{j}+\rho_{j} \xi_{j}^{*}\right)-a=0 .
$$

By the hypothesis that for each pair of functions $f$ and $g$ in $\mathcal{F}, f^{\prime}-a$ and $g^{\prime}-a$ share 0 in $D$, we know that for any positive integer $m$

$$
f_{m}^{\prime}\left(z_{j}+\rho_{j} \xi_{j}\right)-a=0, \quad f_{m}^{\prime}\left(z_{j}+\rho_{j} \xi_{j}^{*}\right)-a=0 .
$$

Fix $m$, take $j \rightarrow \infty$, and note $z_{j}+\rho_{j} \xi_{j} \rightarrow 0, z_{j}+\rho_{j} \xi_{j}^{*} \rightarrow 0$, then $f_{m}^{\prime}(0)-a=0$. Since the zeros of $f_{m}^{\prime}-a$ have no accumulation point, so

$$
z_{j}+\rho_{j} \xi_{j}=0, z_{j}+\rho_{j} \xi_{j}^{*}=0
$$

Hence, $\xi_{j}=-\frac{z_{j}}{\rho_{j}}, \xi_{j}^{*}=-\frac{z_{j}}{\rho_{j}}$. This contradicts with $\xi_{j} \in D\left(\xi_{0}, \delta\right), \xi_{j}^{*} \in D\left(\xi_{0}^{*}, \delta\right)$ and $D\left(\xi_{0}, \delta\right) \cap D\left(\xi_{0}^{*}, \delta\right)=\phi$. Hence, $g^{\prime}-a$ has just a unique zero, which can be denoted by $\xi_{0}$. By Lemma 2.4, $g$ is not any transcendental function.

If $g$ is a nonconstant polynomial, then $g^{\prime}-a=A\left(\xi-\xi_{0}\right)^{l}$, where $A$ is a nonzero constant, $l$ is a positive integer. Thus, $g^{\prime}=A\left(\xi-\xi_{0}\right)^{l}$ and $g^{\prime \prime}=A l\left(\xi-\xi_{0}\right)^{l-1}$. Noting that the zeros of $g$ are of multiplicity $\geq 4$, and $g$ " has only one zero $\xi_{0}$, we see that $g$ has only the same zero $\xi_{0}$ too. Hence, $g^{\prime}\left(\xi_{0}\right)=0$ which contradicts with $g^{\prime}\left(\xi_{0}\right)=a \neq 0$. Therefore, $g$ is a rational function which is not polynomial, and $g^{\prime}+a$ has just a unique zero $\xi_{0}$.

Next, we prove that there exists no rational function such as $g$. Now, we can set

$$
g(\xi)=A \frac{\left(\xi-\xi_{1}\right)^{m_{1}}\left(\xi-\xi_{2}\right)^{m_{2}} \cdots\left(\xi-\xi_{s}\right)^{m_{s}}}{\left(\xi-\eta_{1}\right)^{n_{1}}\left(\xi-\eta_{2}\right)^{n_{2}} \cdots\left(\xi-\eta_{t}\right)^{n_{t}}},
$$

where $A$ is a nonzero constant, $s \geq 1, t \geq 1, m_{i} \geq 4(i=1,2, \ldots, s), n_{j} \geq 2(j=1,2, \ldots, t)$. For stating briefly, denote

$$
m=m_{1}+m_{2}+\cdots+m_{s} \geq 4 s, \quad N=n_{1}+n_{2}+\cdots+n_{t} \geq 2 t .
$$


From (3.3), then

$$
g^{\prime}(\xi)=\frac{A\left(\xi-\xi_{1}\right)^{m_{1}-1}\left(\xi-\xi_{2}\right)^{m_{2}-1} \cdots\left(\xi-\xi_{s}\right)^{m_{s}-1} h(\xi)}{\left(\xi-\eta_{1}\right)^{n_{1}+1}\left(\xi-\eta_{2}\right)^{n_{2}+1} \cdots\left(\xi-\eta_{t}\right)^{n_{t}+1}}=\frac{p_{1}(\xi)}{q_{1}(\xi)}
$$

where

$$
\begin{aligned}
& h(\xi)=(m-N-t) \xi^{s+t-1}+a_{s+t-2} \xi^{s+t-2}+\cdots+a_{0} \\
& p_{1}(\xi)=A\left(\xi-\xi_{1}\right)^{m_{1}-1}\left(\xi-\xi_{2}\right)^{m_{2}-1} \cdots\left(\xi-\xi_{s}\right)^{m_{s}-1} h(\xi), \\
& q_{1}(\xi)=\left(\xi-\eta_{1}\right)^{n_{1}+1}\left(\xi-\eta_{2}\right)^{n_{2}+1} \cdots\left(\xi-\eta_{t}\right)^{n_{t}+1}
\end{aligned}
$$

are polynomials. Since $g^{\prime}(\xi)+a$ has only a unique zero $\xi_{0}$, set

$$
g^{\prime}(\xi)+a=\frac{B\left(\xi-\xi_{0}\right)^{l}}{\left(\xi-\eta_{1}\right)^{n_{1}+1}\left(\xi-\eta_{2}\right)^{n_{2}+1} \cdots\left(\xi-\eta_{t}\right)^{n_{t}+1}},
$$

where $B$ is a nonzero constant, so

$$
g^{\prime \prime}(\xi)=\frac{\left(\xi-\xi_{0}\right)^{l-1} p_{2}(\xi)}{\left(\xi-\eta_{1}\right)^{n_{1}+2}\left(\xi-\eta_{2}\right)^{n_{2}+2} \cdots\left(\xi-\eta_{t}\right)^{n_{t}+2}},
$$

where $p_{2}(\xi)=B(l-N-2 t) \xi^{t}+b_{t-1} \xi^{t-1}+\ldots+b_{0}$ is a polynomial. From (3.5), we also have

$$
g^{\prime \prime}(\xi)=\frac{\left(\xi-\xi_{1}\right)^{m_{1}-2}\left(\xi-\xi_{2}\right)^{m_{2}-2} \cdots\left(\xi-\xi_{s}\right)^{m_{s}-2} p_{3}(\xi)}{\left(\xi-\eta_{1}\right)^{n_{1}+2}\left(\xi-\eta_{2}\right)^{n_{2}+2} \cdots\left(\xi-\eta_{t}\right)^{n_{t}+2}},
$$

where $p_{3}(\xi)$ is also a polynomial.

We use $\operatorname{deg}(p)$ to denote the degree of a polynomial $p(\xi)$.

From (3.5), (3.6) then

$$
\operatorname{deg}(h) \leq s+t-1, \quad \operatorname{deg}\left(p_{1}\right) \leq m+t-1, \quad \operatorname{deg}\left(q_{1}\right)=N+t .
$$

Similarly from (3.8), (3.9) and noting (3.10) then

$$
\begin{aligned}
& \operatorname{deg}\left(p_{2}\right) \leq t \\
& \operatorname{deg}\left(p_{3}\right) \leq \operatorname{deg}\left(p_{1}\right)+t-1-(m-2 s) \leq 2 t+2 s-2 .
\end{aligned}
$$

Note that $m_{i} \geq 4(i=1,2, \ldots, s)$, it follows from (3.5) and (3.7) that $g^{\prime}\left(\xi_{0}\right)=0(i=1$, $2, \ldots, s)$ and $g^{\prime}\left(\xi_{0}\right)=a \neq 0$. Thus, $\xi_{0} \neq \xi_{i}(i=1,2, \ldots, s)$, and then $\left(\xi-\xi_{0}\right)^{l-1}$ is a factor of $p_{3}(\xi)$. Hence, we get that $l-1 \leq \operatorname{deg}\left(p_{3}\right)$. Combining (3.8) and (3.9), we also have $m$ $2 s=\operatorname{deg}\left(p_{2}\right)+l-1-\operatorname{deg}\left(p_{3}\right) \leq \operatorname{deg}\left(p_{2}\right)$. By (3.11), we obtain

$$
m-2 s \leq \operatorname{deg}\left(p_{2}\right) \leq t .
$$

Since $m \geq 4 s$, we know by (3.13) that

$$
2 s \leq t .
$$

If $l \geq N+t$, by (3.12), then

$$
3 t-1 \leq N+t-1 \leq l-1 \leq \operatorname{deg}\left(p_{3}\right) \leq 2 t+2 s-2 .
$$

Noting (3.14), we obtain $1 \leq 0$, a contradiction. 
If $l<N+t$, from (3.5) and (3.7), then $\operatorname{deg}\left(p_{1}\right)=\operatorname{deg}\left(q_{1}\right)$. Noting that $\operatorname{deg}(h) \leq s+t-1$, $\operatorname{deg}\left(p_{1}\right) \leq m+t-1$ and $\operatorname{deg}\left(q_{1}\right)=N+t$, hence $m \geq N+1 \geq 2 t+1$. By (3.13), then $2 t+1 \leq 2 s+t$. From (3.14), we obtain $1 \leq 0$, a contradiction.

The proof of Theorem 1.5 is complete.

Proof of Theorem 1.6 Set $\mathcal{F}^{*}=\left\{\frac{f^{2}}{2} \mid f \in \mathcal{F}\right\}$.

Noting that all zeros of $g \in \mathcal{F}^{*}$ have multiplicities at least 4 and all poles of $g \in \mathcal{F}^{*}$ are multiple, and for each pair of functions $f$ and $g$ in $\mathcal{F}^{*}, f$ and $g^{\prime}$ share a nonzero value $a$ in $D$, we know that $\mathcal{F}^{*}$ is normal in $D$ by Theorem 1.5. Therefore, $\mathcal{F}$ is normal in $D$.

The proof of Theorem 1.6 is complete.

Proof of Theorem 1.7 Set $\mathcal{F}^{*}=\left\{\frac{1}{f} \mid f \in \mathcal{F}\right\}, F:=\frac{1}{f}$.

Noting that $f^{-n} f=-F^{n-2}$ and $n \geq 4$ implies $n-2 \geq 2$, by Theorem 1.4, we know that $\mathcal{F}^{*}$ is normal in $D$.

Since normality of families of $\mathcal{F}$ and $\mathcal{F}^{*}=\left\{\frac{1}{f} \mid f \in \mathcal{F}\right\}$ is the same by the famous Marty's criterion,

Therefore, $\mathcal{F}$ is normal in $D$.

The proof of Theorem 1.7 is complete.

\section{Acknowledgements}

The authors would like to express their hearty thanks to Professor Qingcai Zhang for supplying us his helpful reprint The authors wish to thank the referees and editors for their very helpful comments and useful suggestions. This study was supported partially by the NSF of China (10771220), Doctorial Point Fund of National Education Ministry of China (200810780002).

\section{Author details}

${ }^{1}$ School of Mathematics and Information Science, Guangzhou University, Guangzhou 510006, China ${ }^{2}$ College of Computer Engineering Technology, Guangdong Institute of Science and Technology, Zhuhai 519090, China ${ }^{3}$ School of Economic and Management, Guangzhou University of Chinese Medicine, Guangzhou 510006, China

\section{Authors' contributions}

WY and $J$ carried out the design of the study and performed the analysis. BZ participated in its design and coordination. All authors read and approved the final manuscript.

\section{Competing interests}

The authors declare that they have no competing interests.

Received: 9 June 2011 Accepted: 27 October 2011 Published: 27 October 2011

\section{References}

1. Yang, CC, Yi, HX: Uniqueness Theory of Meromorphic Functions. Science Press, Kluwer Academic Publishers, Beijing, New York (2003)

2. Gu, YX, Pang, XC, Fang, ML: Theory of Normal Family and its Applications (in Chinese). Science Press, Beijing (2007) Hayman, WK: Meromorphic Functions. Clarendon Press, Oxford (1964)

Yang, L: Value Distribution Theory. Springer, Berlin (1993)

Bergweiler, W: Bloch's principle. Comput Methods Funct Theory. 6, 77-108 (2006)

Rubel, LA: Four counterexamples to Bloch's principle. Proc Am Math Soc. 98, 257-260 (1986)

Schwick, W: Normality criteria for families of meromorphic function. J Anal Math. 52, 241-289 (1989)

8. Pang, XC, Zalcman, L: Normal families and shared values. Bull London Math Soc. 32, 325-331 (2000). doi:10.1112/ S002460939900644X

9. Pang, XC, Zalcman, L: Normality and shared values. Ark Mat. 38, 171-182 (2000). doi:10.1007/BF02384496

10. Zhang, QC: Some normality criteria of meromorphic functions. Comp Var Ellip Equat. 53(8), 791-795 (2008). doi:10.1080/ 17476930802124666

11. Hayman, WK: Research Problems of Function Theory. Athlone Press of Univ. of London, London (1967)

12. Gu, YX: Normal Families of Meromorphic Functions (in Chinese). Sichuan Edu. Press, Chengdou (1988)

13. Pang, XC: On normal criterion of meromorphic functions. Sci China Ser. A33, 521-527 (1990)

14. Chen, HH, Fang, ML: On the value distribution of $f^{7} f$. Sci China Ser A. 38, 789-798 (1995)

15. Fang, ML, Zalcman, L: A note on normality and shared value. J Aust Math Soc. 76, 141-150 (2004). doi:10.1017/ S1446788700008752

16. Zalcman, L: A heuristic principle in complex function theory. Am Math Mon. 82, 813-817 (1975). doi:10.2307/2319796 
17. Zalcman, L: Normal families: new perspectives. Bull Am Math Soc. 35, 215-230 (1998). doi:10.1090/50273-0979-98-00755-

18. Bergweiler, W, Pang, XC: On the derivative of meromorphic functions with multiple zeros. J Math Anal Appl. 278, 285-292 (2003). doi:10.1016/50022-247X(02)00349-9

19. Wang, YF, Fang, ML: Picard values and normal families of meromorphic functions with multiple zeros. Acta Math Sin (N. S.). $14(1), 17-26$ (1998). doi:10.1007/BF02563879

20. Bergweiler, W, Eremenko, A: On the singularities of the inverse to a meromorphic function of finite order. Rev Mat Iberoamericana. 11, 355-373 (1995)

doi:10.1186/1029-242X-2011-97

Cite this article as: Yuan et al: Some normality criteria of functions related a Hayman conjecture. Journal of Inequalities and Applications 2011 2011:97.

Submit your manuscript to a SpringerOpen ${ }^{\circ}$ journal and benefit from:

- Convenient online submission

- Rigorous peer review

- Immediate publication on acceptance

- Open access: articles freely available online

- High visibility within the field

- Retaining the copyright to your article

Submit your next manuscript at $\boldsymbol{s p r i n g e r o p e n . c o m ~}$ 